\title{
Avoiding WIMAX Interference on Ultra Wide Band MB- OFDM System by Cognitive Radio
}

\author{
Sarah Fouad Mohamed \\ Modern Academy
}

\author{
Yasmine Fahmy \\ Cairo University
}

\author{
Abd El Halim Zekry \\ Ain Shamis University
}

\begin{abstract}
This paper considers the ECMA-368 standard based on Multiband Orthogonal Frequency Division Multiplexing (MB-OFDM) as an Ultra Wideband (UWB) system in the presence of interference from an IEEE 802.16 WiMAX systems operating at $3.5 \mathrm{GHz}$. Simulations are conducted following the standards and adopting the IEEE 802.15.3a channel model CM1. This paper shows that the system fails because of the WiMAX interference, in the absence of an interference avoidance or cancellation scheme. This paper exploits the channel information and the flexibility of noncontiguous Orthogonal Frequency Division Multiplexing (NC-OFDM) based cognitive radios to avoid coexistence interference between UWB and WiMAX systems. The proposed cognitive UWB system results in a significant gain and is compatible with minimum changes to the current system specifications.
\end{abstract}

Index Terms- Coexistence interference, MB-OFDM, UWB, WiMAX

\section{INTRODUCTION}

This paper considers the ECMA-368 Multiband Orthogonal Frequency Division Multiplexing (MB-OFDM) standard for high rate Ultra-Wideband (UWB) wireless communications in the 3.1-10.6 GHz band $[1,2]$. Since UWB systems in this band are operating as spectral underlay systems $[3,4]$, they will unavoidably be impacted by the transmission of incumbent systems. We consider as interferer the WiMAX IEEE 802.16 system for wireless Metropolitan Area Networks; operating in the licensed 3.5 GHz band [5]. Both modulation techniques included in the WiMAX standard: Single Carrier (SC) and OFDM for use below $11 \mathrm{GHz}$ are addressed in this paper.

There has recently been great interest in coexistence techniques between WiMAX and UWB systems [6, 7]. The authors of [7] considered the effect of WiMAX interference signals on MB-OFDM UWB systems for high rate. They derived the exact expression of the uncoded bit error rate (BER) of the MB-OFDM system based on a Laplace transform technique. However they did not consider the Coded OFDM based UWB systems as a victim receiver in the presence of the modified Saleh-Valenzuela model [8] which is adopted to be used as a reference UWB channel by IEEE 802.15.3a study group.

In [10], the sub carriers corrupted by notch band interference are considered as unreliable, they are discarded in demodulation, and the lost data due to frequency domain excision can be recovered with the help of channel coding method.

Where as in [11], the per-sub carrier interference plus noise power is estimated and used to weigh the branch metrics fed to the Viterbi decoder in order to suppress the interference effects. This particular technique requires only modest increases in receiver complexity and does not require any modifications to the MB-OFDM transmitter or signal structure.

In contrast to $[6,9]$, we suggest to avoid coexistence interference due to a WiMAX system on the MB-OFDM UWB system by deactivating (i.e. nulling) subcarriers that are potentially interfered by the other system. This is done by applying the concept of cognitive radio system and using the channel information at the transmitter side.

In the next sections the used simulation model consisting of the MB-OFDM UWB system [1, 2], the WiMAX system modeled as colored Gaussian distribution interference are briefly explained. Then the proposed non-contiguous OFDM (NC-OFDM) [9] as an interference avoidance technique is demonstrated in section 3. Finally, the simulation results for the system performance are summarized in section 4 over various interference bandwidths and NC-OFDM as an interference avoidance technique over the CM1 channel model [6]. Then the paper is concluded in the final sections.

\section{SYSTEM MODEL}

\subsection{MB-OFDM signal model for UWB}

For high data rate UWB application, performance, complexity and system flexibility are the like criteria. The performance of the UWB system is determined by its robustness to multipath channel environments, ability to handle narrow band interferers and other UWB interferers. This leads to make OFDM as a suitable modulation technique for UWB system. The transmitted RF signal can be written in terms of the complex baseband signal as follows

$r_{R F}(t)=\operatorname{Re}\left\{\sum_{k=0}^{N-1} r_{k}\left(t-k T_{S Y M}\right) \exp \left(J 2 \pi f_{(k \bmod 6)} t\right)\right.$

Where Re (.) represents the real part of a complex variable, $r_{k}(t)$ is the (possibility complex) base band signal representing the $k^{\text {th }}$ OFDM symbol occupying a symbol intervals of length $T_{S Y M}$, and $\mathrm{N}$ is the number of OFDM symbols transmitted, the carrier frequency or band that the $k^{t h}$ OFDM symbol is transmitted over is denoted as $f_{k}$. The values of $f_{k}$ range over 3 frequencies assigned to the band group. These frequencies are organized into sequences of length 6 , called time-frequency codes (TFCs).

The MB-OFDM UWB system (as shown in Fig.1.) is described as the convolution encoder shall use the rate $\mathrm{R}=1 / 3$ code. Puncture is a procedure for omitting some of the encoded bits at the transmitter. Here we omit the second bit of the encoded bits at the transmitter but at the decoder we insert a dummy "zero" metric at the receiver in place of the second omitted bits. The coded and padded bit stream should be interleaved prior to modulation to provide robustness against burst errors. The coded and interleaved binary serial input data will be divided into groups of two bits and converted into a complex number representing one of the four Qpsk 
constellation points. Each OFDM symbol is converted to the time domain using a 128 point Inverse Fast Fourier Transform (IFFT), and each OFDM symbols are groups of 100 data symbols, 12 pilots, 6 zeros padded, and 10 guard sub carriers. A guard interval of time domain $\mathrm{T}_{\mathrm{g}}=70.7 \mathrm{~ns}$ (37 samples) is appended to each OFDM symbol before transmission. The transmitted MB-OFDM symbol occupies a bandwidth of 528 MHZ. The standard employs frequency-hopping techniques in which the carrier frequency of MB-OFDM transmission is changed after each OFDM symbol .
Where the impulse response of the channel is $h(t), i(t)$ is the interference, and $n(t)$ is the complex additive white Gaussian noise (AWGN).

As shown in [7], since the MB-OFDM systems hops over three bands, the interference power in two of these bands is zero, the overall average SIR is given by

$$
S I R=\frac{3 E_{b}}{2 N_{i}}
$$
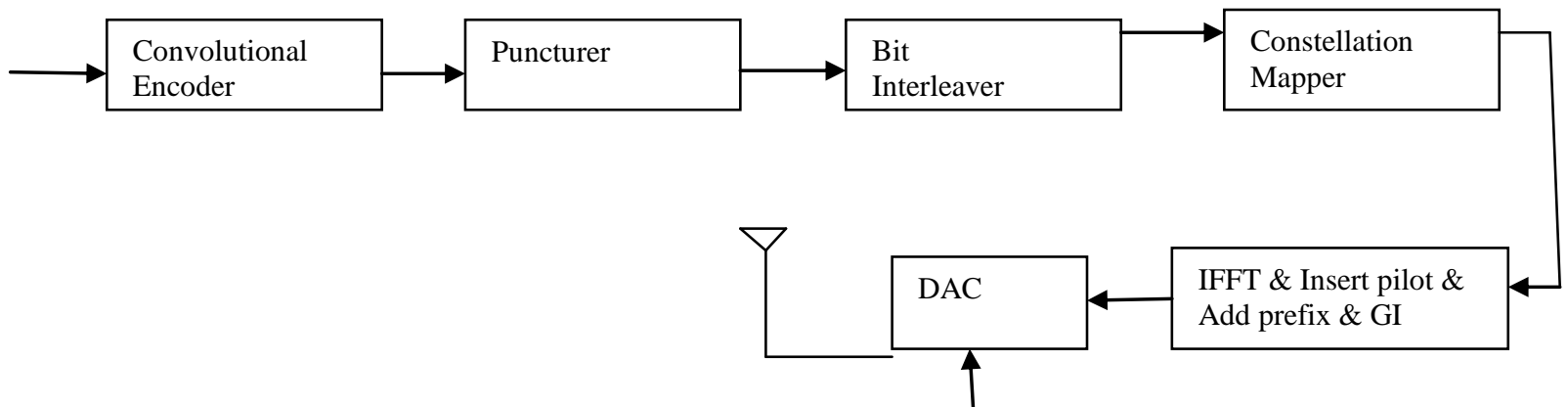

Time frequency kernel

Fig.1. Transmitter architecture for MB-OFDM UWB system.

\subsection{WiMAX Interference Signal Model}

The WiMAX standard [5, 7] applies two types of modulations with different bandwidths. The WiMAX-OFDM transmitted signal is given by

$$
S_{n}(t)=\sum_{l=-\infty}^{\infty} \sum_{d=0}^{N_{n}-1} Z_{d, l} \theta_{d}\left(t-l T_{n}\right) e^{j 2 \pi f_{n} t}
$$

Where $N_{n}$, and $f_{n}$ are the numbers of sub carrier, the center frequency, while $Z_{d, l}$, and $\theta_{d}(t)$ are the modulated symbols, and the basis function for sub carriers.

On the other hand the WiMAX-SC transmitted signal is given by

$$
S_{S}(t)=\sum_{l=-\infty}^{\infty} Z_{l} p\left(t-l T_{S}\right) e^{j 2 \pi f_{s} t}
$$

Where $Z_{l}, f_{s}$, and $T_{s}$ are the modulated symbols, WiMAX-SC carrier frequency, and WiMAX-SC symbol period. $p(t)$ denotes the square-root raised cosine pulse shaping filter with roll-off factor 0.25 .

Due to small bandwidth of WiMAX systems both OFDM and SC modulation compared to the UWB system, the WiMAX appears as tone interference to the UWB system. We simulated the WiMAX signal as a colored Gaussian distribution [12] which is a Gaussian distribution followed by a raised cosine filter.

The received signal at the receiver of the UWB MB-OFDM system can be expressed by

$$
r(t)=\left[s_{m}(t) \otimes h(t)\right] \exp \left(j 2 \pi f_{(k \bmod 6)} t\right)+i(t)+n(t)
$$

\subsection{Simulation Interference Model}

As a WiMAX released in different center frequencies we focus on the $3.5 \mathrm{GHZ}$ center frequency, as a single carrier modulated signal or as a multicarrier OFDM system. In both cases the bandwidth of the WIMAX interference signal may occupy different band widths. Therefore, the effect of the interfering signal bandwidth will be taken into account on the UWB performance. In addition, the effect of the interfering signal strength, expressed as the PSD of WiMAX signal on the UWB system must be considered. The most suitable interfering signal model for such WIMAX signal is the colored Gaussian noise (CGN), because the spectral allocation and the power level for the interference could independently be selected based on the central limit theorem [12].

This is why a band-limited CGN model was selected as the general interference model. For the spectrum allocation, both the center frequency and bandwidth can be defined. In this case, the white Gaussian noise signal is passed through a raised cosine filter. The output, after the filtering, has colored PSD as the name obviously indicates.

\section{Non-Contiguous OFDM for UWB system}

Cognitive radios have been advanced as a technology for the opportunistic use of underutilized spectrum wherein secondary devices senses the presence of the primary user and use the spectrum only if it is empty. An example of this as shown in the following is the operation of UWB devices in WiMAX bands. UWB as a secondary user must avoid WiMAX devices in certain regulatory domains.

Multi Carrier Modulation (MCM) is highly suited for high speed data transmission, due to its ability to efficiently handle the distortion introduced by frequency selective channels. 
OFDM as a MCM technique can provide the necessary agile spectrum usage, when portions of the target licensed spectrum are occupied by both primary and secondary users. This achieve by deactivating (i.e. nulling) subcarriers that can interfere with other users [9].

This technique is known as NC-OFDM as a modulation technique can be used instead of OFDM modulation in UWB system.

From the above cognitive radio definition one natural and near-optimal solution for interference avoidance can be accomplished in two steps;

1- The UWB secondary user collects measurement information and makes decisions, on the granting portions of the spectrum.

2- The sub carriers corresponding to the spectrum occupied by incumbent primary user transmission which are determined from the spectrum sensing measurement are deactivated by NC-OFDM.

Figure 2 shows the block diagram of the ECMA 368 standard UWB system operating as a cognitive radio system by adding NULL Sub carrier selection block. The remaining blocks are ordinary digital signal processing ones comprising channel coding, and the OFDM building blocks.

The following blocks follow the ECMA368 standard instead that after Qpsk block the modulated data stream is then split into $\mathrm{N}$ slower data streams using a serial-to-parallel (S/P) converter. Note that the sub carriers in the NC-OFDM transceiver do not need to be all active as in conventional OFDM transmission. Moreover, the active subcarriers are located in the unoccupied spectrum bands, which are determined by Dynamic Spectrum Sensing and channel estimation techniques. The inverse Fast Fourier transform (IFFT) converts the OFDM symbol from frequency domain to time domain. The output of the IFFT block as shown in for the $\mathrm{q}_{\mathrm{th}} \mathrm{NC}-\mathrm{OFDM}$ symbol is given by

$$
Y_{k}=\sum_{k=0}^{N-1} r_{k}\left(t-k T_{S Y M}\right)
$$

where $r_{k}$ the symbol of the $\mathrm{k}_{\mathrm{th}}$ subcarrier but the symbol is over the $\mathrm{k}_{\mathrm{th}}$ deactivated sub carrier is $\mathrm{r}_{\mathrm{k}}=0$ Prior to transmission, a guard interval of length greater than the channel delay spread is added to each NC-OFDM symbol known as cyclic prefix (CP) following parallel-to-serial conversion.
The base band NC-OFDM signal is then passed through the transmitter radio frequency (RF) chain, which amplifies the signal and up converts it into the desired center frequency following the time frequency code. The receiver performs the reverse operation of the transmitter.

\subsection{NC-OFDM signal-to-Noise Ratio analysis}

The SNR is defined as the ratio of the desired signal power to the noise power. The SNR indicates reliability of transmission link between the transmitter and receiver, and is accepted as a standard measure of signal quality.

For NC-OFDM system as shown in [9], considered an AWGN channel with noise spectral density $\mathrm{N}_{0}$ and bandwidth $\mathrm{B}$, the noise power is given by:

While the SNR is given by:

$$
E\left(\left|\widetilde{n_{1}}\right|=\sigma_{N}^{2}=N_{0} B\right.
$$

$$
\gamma_{1}=10 \log _{10}\left(\frac{E\left|x_{1}\right|^{2}}{\sigma_{N}^{2}}\right)=10 \log _{10}\left(\frac{E\left|x_{1}\right|^{2}}{N_{0} B}\right)
$$

Suppose the incumbent spectral occupancy (ISO) is $\alpha$, then the total available bandwidth would be $(1-\alpha) B$ since the channel response is assumed to be approximately flat, the signal power would remain almost constant, irrespective of the available bandwidth. However, the effective noise power would be:

$$
\sigma_{N}^{2}=N_{0}(1-\alpha) B
$$

Then, the SNR is given as follows

$$
\gamma_{2}=10 \log _{10}\left(\frac{E\left|x_{1}\right|^{2}}{\sigma_{N}^{2}}\right)=10 \log _{10}\left(\frac{E\left|x_{1}\right|^{2}}{N_{0}(1-\alpha) B}\right)
$$

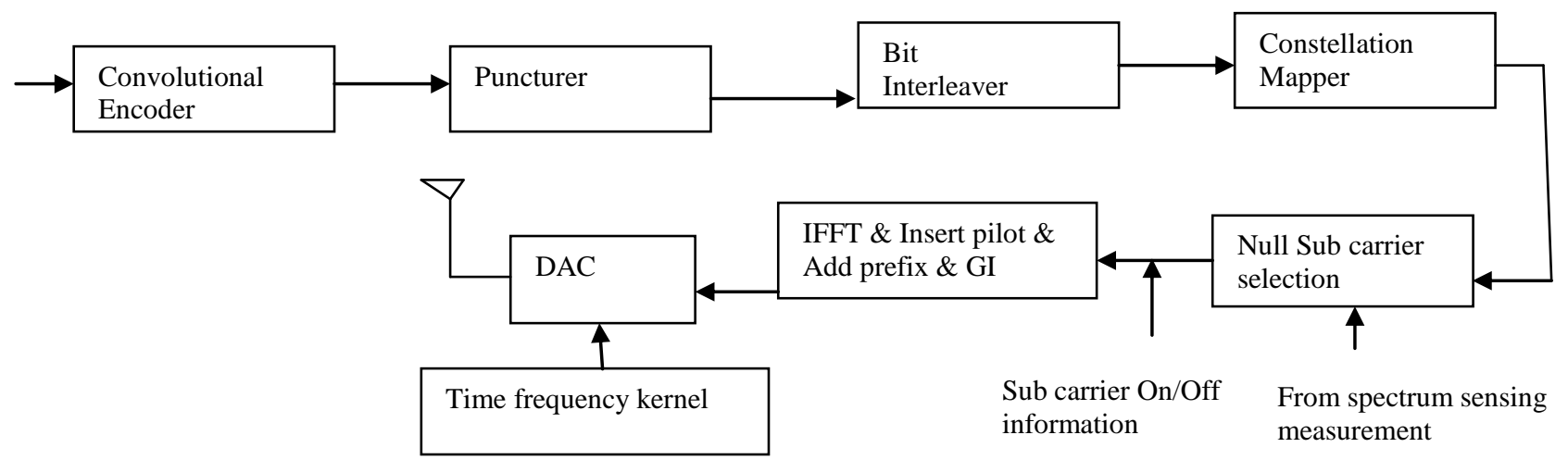

Fig.2. ECMA368 standard with NC-OFDM 


\section{SIMULATION RESULTS}

The above UWB system with NC-OFDM to avoid the strong WiMAX primary interferer is implemented using Matlab code to evaluate its performance. The system is built according to the ECMA 368 standard [1, 2] for coded MB-OFDM system at $320 \mathrm{Mbps}$ to the system parameters shown in Table. 1.

For each transmitted frame, a different realization of the UWB channel model CM1, specified by the IEEE802.15.3a channel modeling subcommittee report and described in [8], has been carried out.

Figure 3 compares the performance of MB-OFDM UWB System under the CM1 channel model without interference and with interference at different bandwidths of the interfering WiMax system of 7 and 17.5 MHZ. The signal to interference power ratio SIR is assumed to be $-10 \mathrm{~dB}$. From this Figure we see that bit error rate BER is degraded significantly and becomes unacceptable even at high Signal to Noise ratio (SNR) as it exceed $10^{-1}$.

Figure 4, shows the simulated bit error rate as a function of the signal to interference ratio SIR at a SNR of $8 \mathrm{~dB}$. Again, different interference bandwidths are considered; 3.5, 7 and 17.5 MHz. It is clear from this figure that as SIR increases the bit error rate decreases for all values of interferer band width. When the SIR reaches about $10 \mathrm{~dB}$ the BER becomes clamped at 0.0040 .

Results for similar simulations are shown in Figure 5 for the SC modulation WiMAX system with $12.5,25$ and $50 \mathrm{MHz}$ bandwidths. The performance of this system is similar to the previous system.

The simulation results in Figure 6 and 7 compare the performance of the MB-OFDM without interference, with $\{7$, and 3.5 $\mathrm{MHz}$ bandwidth interference and the cognitive interference avoidance technique of NC-OFDM. While the system under interference suffers from an error floor, the NCOFDM system performance does not clearly suffer from such an effect. The penalty of this significant gain is a lower bit rate $(243 \mathrm{Mbps})$ due to the deactivation of $27 \%$ of the subcarriers in the first sub band.

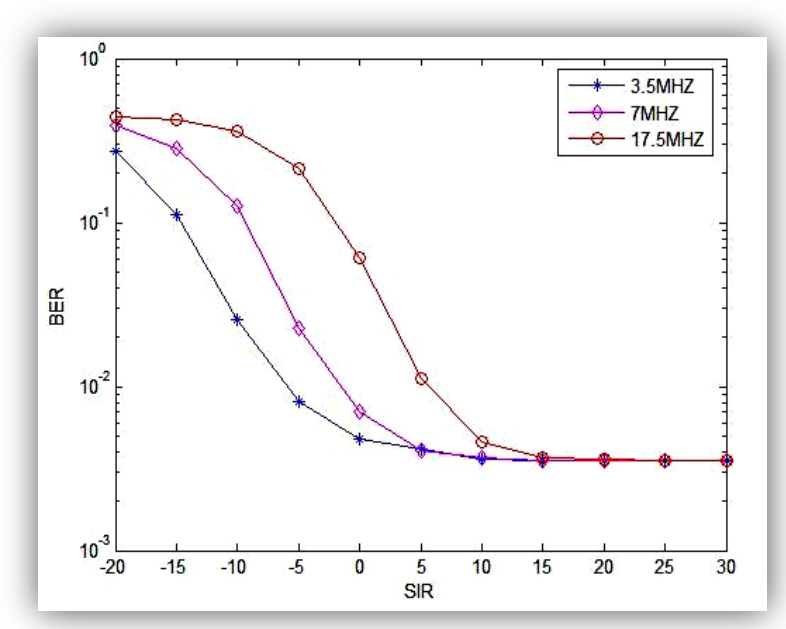

Fig.3.MB-OFDM BER without and with WiMAX interference of $-10 \mathrm{~dB}$ level at $\{7,17.5\} \mathrm{MHz}$ bandwidth.

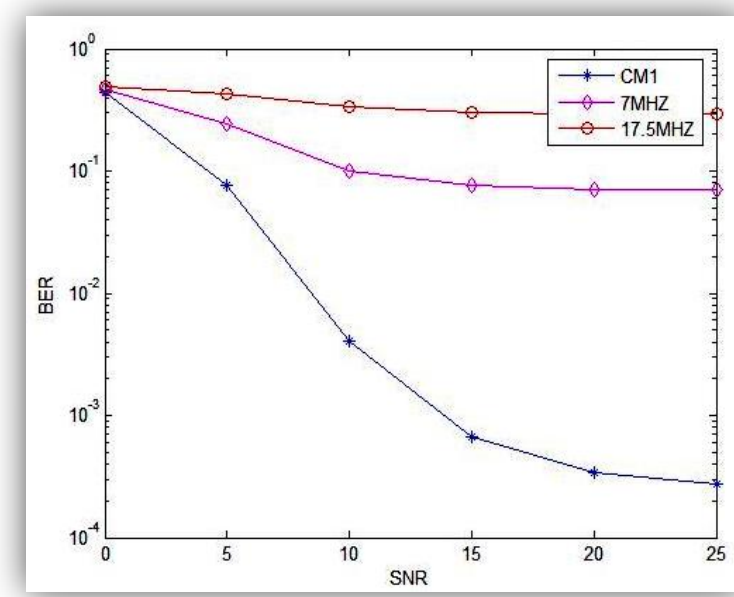

Fig.4.MB-OFDM BER versus SIR with 8dB SNR and WiMAX-OFDM bandwidth $\{3.5,7,17.5\} \mathrm{MHz}$.

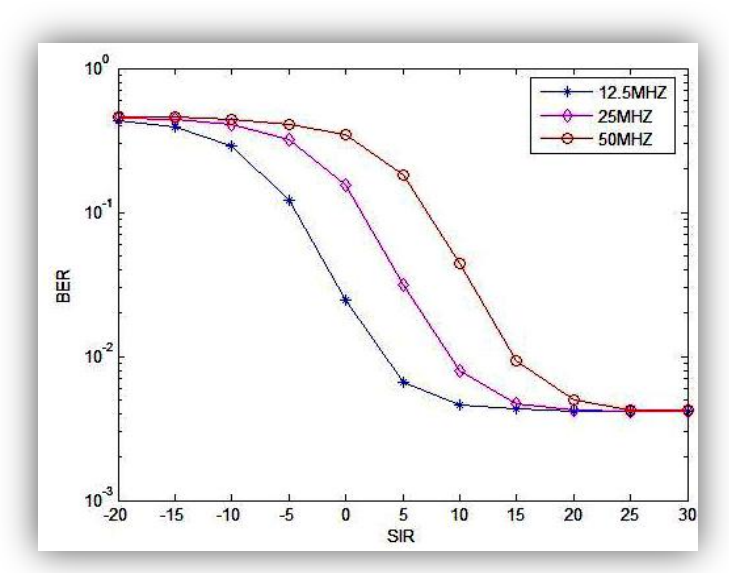

Fig.5. MB-OFDM BER versus SIR with SNR of $8 \mathrm{~dB}$ and WiMAX-SC bandwidths $\{12.5,25,50\} \mathrm{MHz}$.

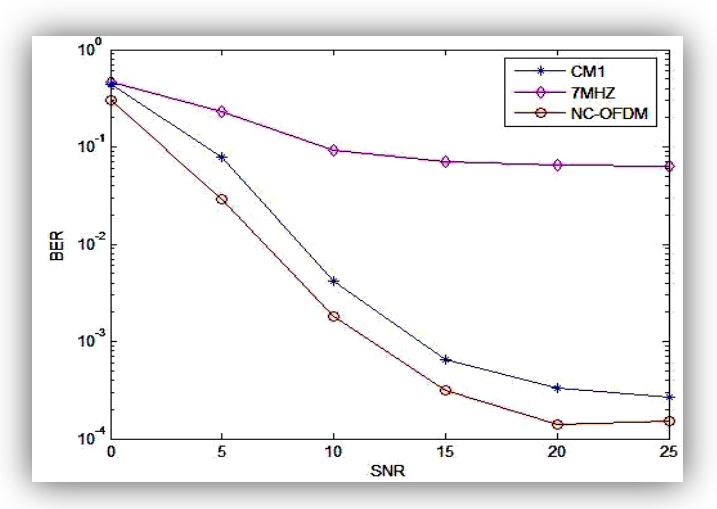

Fig.6. MB-OFDM BER without and with $7 \mathrm{MHZ}$ interference compared to the proposed NC-OFDM as cognitive radio. 


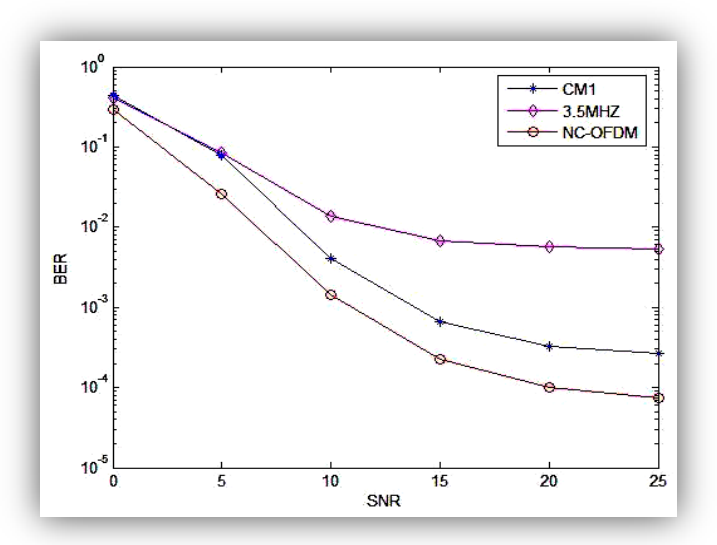

Fig.7.MB-OFDM BER without and with $3.5 \mathrm{MHz}$ interference compared to the proposed NC-OFDM as cognitive radio.

Table.1 Simulation parameters

\begin{tabular}{|c|c|c|}
\hline Parameters & Description & Value \\
\hline Bit rate & $\begin{array}{l}\text { Bit rate "channel } \\
\text { coding" }\end{array}$ & $\begin{array}{c}320 \\
\text { Mbps }\end{array}$ \\
\hline$R_{c}$ & Coding Puncturing rate & $1 / 2$ \\
\hline$w_{m}$ & Bandwidth of sub band & $\begin{array}{c}528 \\
\mathrm{MHZ}\end{array}$ \\
\hline$N_{m}$ & $\begin{array}{l}\text { Total number of sub } \\
\text { carriers (FFT size) }\end{array}$ & 128 \\
\hline$N_{D}$ & $\begin{array}{c}\text { Number of Data sub } \\
\text { carriers }\end{array}$ & 100 \\
\hline$N_{p}$ & Number of sub carriers & 12 \\
\hline$N_{G}$ & $\begin{array}{l}\text { Number of guard sub } \\
\text { carriers }\end{array}$ & 10 \\
\hline$N_{T}$ & $\begin{array}{c}\text { Total number of sub } \\
\text { carriers used }\end{array}$ & 122 \\
\hline$Q_{m}$ & $\begin{array}{l}\text { Sub-carrier frequency } \\
\text { spacing }\end{array}$ & $\begin{array}{l}4.125 \\
\text { MHZ }\end{array}$ \\
\hline$D_{m}$ & IFFT and FFT period & $\begin{array}{c}242,42 \\
\text { ns }\end{array}$ \\
\hline$C_{m}$ & $\begin{array}{l}\text { Zero-padded suffix } \\
\text { duration in time }\end{array}$ & $\begin{array}{c}70,08 \\
\text { ns }\end{array}$ \\
\hline$T_{m}$ & Symbol interleaving & $\begin{array}{c}312,5 \\
\mathrm{~ns}\end{array}$ \\
\hline CM1 & UWB channel & 100 \\
\hline
\end{tabular}

\section{CONCLUSION}

This paper shows great performance degradation of the UWB based on ECMA 368 standard $[1,2]$ for coded MB-OFDM when interfered by the WiMAX signal operating at $3.5 \mathrm{GHZ}$ center frequency. This is because the WiMAX is strong primary signal while the UWB system of a weak secondary signal. We proved that the use of cognitive NC-OFDM is an effective and straight forward technique to avoid coexisting interference from strong WiMAX signal on the very weak UWB signal. The performance gains of this technique greatly overweigh the system loss arising from rate reduction due to deactivation of sub-carriers in the first sub-band.

\section{REFERENCES}

[1] ECMA, "Standard ECMA-368: High Rate Ultra Wideband PHY and MAC Standard," Dec. 2007.

[2] A. Batra, J. Balakrishnan, G. Aiello, J. Forester, and A. Dabak, "Design of a Multiband OFDM System for Realistic UWB Channel Environments," IEEE Trans. Microwave Theory Tech., vol. 52, no. 9, pp. 2123-2138, Sept. 2004.

[3] Federal Communications Commission (FCC), "Revision of Part 15 of the Commissions Rules Regarding UltraWideband Transmission Systems," First Report and Order, ET Docket 98-153, FCC 02-48; Adopted: February 14, 2002; Released: April 22, 2002.

[4] Q. Zhao and B. M. Sadler, "A Survey of Dynamic Spectrum Access," IEEE Signal Processing Mag., vol.24, no. 3, pp. 79-89, May 2007.

[5] IEEE Std 802.16-2004, "Part 16: Air Interface for Fixed Broadband Wireless Access Systems," Oct. 2004.

[6] V. Somayazulu, J. Foerster, and R. Roberts, "Detect and Avoid (DAA) Mechanisms for UWB Interference Mitigation," in Proc. IEEE Intl. Conf. on Ultra-Wideband (ICUWB), Waltham, MA, USA, Sept. 2006, pp. 513518.

[7] C. Snow, L. Lampe, R. Schober "Analysis of the impact of WiMaX-OFDM interference on Multiband OFDM' In Proc. IEEE International conference on Ultra-Wideband, Singapore, September 2007.

[8] A. F. Molisch, J. R. Forester, and M. Pendergrass, "Channel Models for Ultra Wideband Personal Area Networks," IEEE wireless Commun, Mag., PP. 14-21, Dec. 2003.

[9] R. Rajbanshi, A. M. Wyglinski, and G. J. Minden, "OFDM-Based Cognitive Radios for Dynamic Spectrum Access Networks", springer chapter 2007.

[10] K. Shi, B. Kelleci, T. W. Fischer, Y. Zhou, E. Serpedin, and A. Karsilayan, "On the design of robust multiband OFDM ultra-wideband receivers" presented at the 2005 Texas Wireless Symp., University of Texas at Austin, Austin, TX

[11] C. Snow, L. Lampe, and R. Schober. "Interference Mitigation for coded MB-OFDM UWB," In Proc. IEEE Radio and Wireless Symposium Orlando, Fl, USA, January 2008. Invited paper.

[12] Lloyd Emmanuel, Xavier .N. Fernando "Wavelet based spectral shaping of UWB radio signal for multi system coexistence " Science Direct Computers \& Electrical Engineers, Vol. 36, Issue 2, pp. 261-268, March 2010.

[13] 12- G. Caire, G. Taircco, and E. Biglieri. Bit-Interleaved Coded Modulation. IEEE Trans. Inform. Theory, vol. 44, no. 3, pp. 927-946, May 1998. 